\title{
Desenvolvimento psicomotor de meninos e meninas com 8 anos de idade*
}

\section{Psychomotor development of 8 years old boys and girls}

Ester Pereira Lages ${ }^{1}$ Fernanda Barbosa Alvarenga ${ }^{2}$ Renata Aparecida Elias Dantas ${ }^{3}$
Recebido em: 15/09/2016. Aprovado em: 19/01/2017.

1 Graduando em Educação Física - Centro Universitário de Brasília - UniCEUB - Brasília.

2 Graduanda em Educação Física - Centro Universitário de Brasília - UniCEUB - Brasília.

3 Professora Doutora - Centro Universitário de Brasília - UniCEUB - Brasília.

\section{Resumo}

Sabe-se que o desenvolvimento motor do ser humano é um assunto muito estudado, inúmeras são as pesquisas realizadas com a finalidade de avaliar as fases motoras evolutivas correspondentes à sua maturação biológica. O objetivo deste estudo foi avaliar o nível de desenvolvimento motor e fazer uma comparação entre os sexos de crianças de um colégio em Brasília-DF. Aplicou-se o teste KorperkoordinationstestfurKinder (KTK), para avaliar o desenvolvimento motor das crianças de oito anos de idade e de ambos os sexos, que praticam aulas de educação física. O teste foi constituído de quatro tarefas: Trave de equilíbrio, saltos monopedais, saltos laterais e transferência sobre plataformas. Os resultados apontaram diferença significativa entre os sexos quanto à habilidade equilíbrio $(\mathrm{p}=$ 0,002 ). Conclui-se que, em relação a análise por sexo não houve diferença significativa nos aspectos motores quanto à força, velocidade e lateralidade ( $\mathrm{p}=0,756 ; \mathrm{p}=1,000$ e $\mathrm{p}=1,000$ respectivamente).

Palavras-chave: Desenvolvimento psicomotor. Crianças. Escolares. Educação Física.

\begin{abstract}
It is well known that the motor development of the human being has been the subject of extensive research throughout history. Many studies have sought to evaluate the evolutionary motor phases in correlation with biological maturation. The purpose of this study was to evaluate and compare by gender the level of motor development of children in a school in Brasilia, DF. The KorperkoordinationstestfurKinder (KTK) test was used to assess the motor development of 8-year old children of both genders, take physical education classes. The test consisted of four subtests: traversing a balance beam, hopping on one foot, jumping laterally, and moving sideways on platforms. The results showed significant differences between genders regarding balance $(\mathrm{p}=0.002)$. We concluded that motor aspects such as strength, speed, and lateral movement showed no significant differences when evaluated by gender $(\mathrm{p}=0.756 ; \mathrm{p}=1.000$ and $\mathrm{p}=1.000$ respectively).
\end{abstract}

Keywords: Psychomotor development. Children. Physically active. 


\section{Introdução}

Sabe-se que o desenvolvimento motor do ser humano é um assunto muito estudado ao longo da história, muitas pesquisas foram realizadas com a finalidade de avaliar as fases motoras evolutivas correspondentes a sua maturação biológica. Cada criança tem sua fase de desenvolvimento pré-determinada, porém, é preciso dar estímulos suficientes para que as habilidades motoras se desenvolvam dentro do tempo esperado, tais estímulos são dados no dia a dia com brincadeiras, jogos e nas aulas de Educação física. (WEINECK, 1986; MEINEL; SCHNABEL, 1988)

$\mathrm{Na}$ atualidade, é cada vez maior a convivência com a tecnologia, tais como: tablets computadores, jogos eletrônicos etc. Estes, por sua vez, estão "roubando" o precioso tempo das crianças de brincar na rua, correr, chutar, arremessar, jogar, pegar e demais atividades que estimulam o aprendizado motor, movimentos que são essenciais para desenvolver as habilidades básicas das crianças nas fases inicias. As referidas habilidades são requisitadas na vida diária da criança, tanto na escola como em casa, a fim de atender aos seus diversos propósitos. Essas habilidades, denominadas de habilidades motoras básicas, são vistas como o alicerce para a aquisição de habilidades motoras especializadas, na dimensão artística, esportiva, ocupacional ou industrial (TANI et al., 1988).

Adquirindo capacidade de coordenação motora educação física tem um papel fundamental na vida das crianças, pois o professor tem o dever de incentivar, por meio de jogos, brincadeiras e atividades lúdicas, o desenvolvimento motor nas séries iniciais.

Percebe-se que, por um lado, as práticas corporais observadas nas aulas de Educação Física, no contexto escolar, estão focadas no movimento humano de forma consciente, por outro lado, se faz necessária a pesquisa em torno de como está acontecendo o desenvolvimento do esquema corporal das crianças. Sendo assim, necessi- ta-se de mais compreensão e entendimento no contexto de como os professores de Educação Física estão trabalhando o desenvolvimento das habilidades motoras nas aulas de educação física.

A criança, por meio da observação, imitação e experimentação das instruções recebidas de pessoas mais experientes, vivencia diversas experiências físicas e culturais, construindo, dessa forma, o conhecimento a respeito do mundo que a cerca (BONAMIGO et al., 1982).

O objetivo desse estudo foi avaliar o nível dos aspectos psicomotores de crianças de ambos os sexos de oito anos de idade, 10 meninos e 11 meninas praticantes de aulas de educação física.

\section{Materiais e Métodos}

Essa pesquisa trata de um estudo transversal de caráter exploratório. Para isso o projeto foi submetido ao Comitê de Ética do Centro universitário de Brasília, sendo aprovado com CAAE: 52865715.0.0000.0023 e Número do Parecer: 1.408.293. Seguindo a resolução 466-12 para pesquisas realizadas com seres humanos.

\subsection{Amostra}

Participaram da pesquisa 11 meninas e 10 meninos de oito anos de idade.

\subsection{Métodos}

Foi aplicado o teste KTK (Korperkoordinationstest fur Kinder), desenvolvido por Kiphard e Schilling (1974), com o intuito de analisar o desenvolvimento motor das crianças que participam das aulas de educação física. A bateria foi composta pelas etapas descritas abaixo.

\subsubsection{Saltos Laterais (SL)}

Objetivo: Velocidade em saltos alternados.

Material: Uma plataforma de madeira (compensado) de $60 \times 50 \times 0,8 \mathrm{~cm}$, com um

sarrafo divisório de 60 × 4 × $2 \mathrm{~cm}$ e um cronome-

\subsubsection{Transferência sobre plataforma (TP)}

Objetivo: lateralidade; estruturação espaço temporal.

Material: Um cronometro e duas plataformas de madeira com $25 \times 25$ x $1,5 \mathrm{~cm}$ e em cujas esquinas se encontram aparafusados quatro pés com 3,5 com de altura, tal como figura 10. Na direção de deslocar, foi necessário uma área livre de 5 a 6 metros . 


\subsubsection{Trave de Equilíbrio (EQ)}

Objetivo: estabilidade do equilíbrio em marcha para trás sobre a trave.

Material: Foram utilizadas três traves de 3 metros de comprimento e $3 \mathrm{~cm}$ de altura, com larguras de $6 \mathrm{~cm}$, $4,5 \mathrm{~cm}$ e $3 \mathrm{~cm}$. Na parte inferior, são presos pequenos travessões de $15 \mathrm{x}, 5 \mathrm{x} 5 \mathrm{~cm}$, espaçados de $50 \mathrm{em} 50 \mathrm{~cm}$. Com isso, as traves alcançam uma altura total de $5 \mathrm{~cm}$. Como superfície de apoio para saída, coloca-se, em frente à trave, uma plataforma medindo $25 \times 25 \times 5 \mathrm{~cm}$. As três traves de equilíbrio são colocadas paralelamente.

\subsubsection{Saltos Monopedais (SM)}

Objetivo: Coordenação dos membros inferiores; energia dinâmica/forca.

Material: São usados 3 blocos de espuma, medindo cada um $50 \mathrm{~cm} \times 20 \mathrm{~cm} \times 5 \mathrm{~cm}$.

\subsection{Análise Estatística}

Os dados foram analisados utilizando o software SPSS versão 21 (SPSS Inc., Chicago, IL, EUA). A análise descritiva dos dados foi realizada e expressa nas tabelas em média e desvio padrão. A normalidade dos dados foi verificada pelo teste de Shapiro-Wilk. Como a normalidade dos dados não foi confirmada, a comparação entre os escores do sexo masculino e do sexo feminino foi realizada por meio do teste $\mathrm{U}$ de Mann-Whitney. O nível de significância estatística adotado foi de $\mathrm{p}<0,05$.

\section{Resultados}

A descrição dos escores em cada sexo está exposta em média e desvio padrão na tabela 1. Após a realização do teste não paramétrico, os resultados apontaram diferença significativa entre os sexos na trave de equilibrio $(\mathrm{p}=0,002)$. Nesse sentido, crianças do sexo masculino mostraram um escore, significativamente, superior que as crianças do sexo feminino.

Entretanto, os escores do Salto Lateral, Transferência sobre Plataforma e Salto Monopodal não se diferiram significativamente $(\mathrm{p}=0,756 ; \mathrm{p}=1,000$ e $\mathrm{p}=1,000$ respectivamente), inclusive apontando mesmo escore entre os sexos tanto na Transferência sobre Plataforma quanto no Salto Monopodal.

Tabela 1 - Análise descritiva dos escores e diferença entre sexo.

\begin{tabular}{|c|c|c|c|c|c|c|c|}
\hline Variável & Sexo & $\mathbf{n}$ & m & $d p$ & med & aiq & $\mathbf{p}$ \\
\hline \multirow{2}{*}{ Trave de Equilíbrio } & Masculino & 10 & 69,00 & 6,32 & 72,00 & 3,75 & \multirow{2}{*}{0,002} \\
\hline & Feminino & 11 & 43,82 & 16,92 & 41,00 & 29,00 & \\
\hline \multirow{2}{*}{ Salto Lateral } & Masculino & 10 & 15,10 & 6,31 & 18,00 & 8,00 & \multirow{2}{*}{0,756} \\
\hline & Feminino & 11 & 20,55 & 16,64 & 17,00 & 36,00 & \\
\hline \multirow{2}{*}{ Transferência de Plataforma } & Masculino & 10 & 15,00 & 0,00 & - & - & \multirow{2}{*}{1,000} \\
\hline & Feminino & 11 & 15,00 & 0,00 & - & - & \\
\hline \multirow{2}{*}{ Salto Monopodal } & Masculino & 10 & 0,00 & 0,00 & - & - & \multirow{2}{*}{1,000} \\
\hline & Feminino & 11 & 0,00 & 0,00 & - & - & \\
\hline
\end{tabular}

$\mathrm{N}$ : tamanho da amostra; M: media; DP: desvio padrão; Med: mediana; AIQ: amplitude interquartil; p: diferenças analisadas pelo teste de U de Mann-Whitney Fonte: autoria própria.

\section{Discussão}

Este estudo teve por objetivo avaliar o nível psicomotor de crianças de 8 anos de idade de ambos os sexos. No estudo realizado por Da Cruz (2012), com 19 crianças na faixa etária de 8 anos, analisando por sexo, e utilizando o mesmo teste (KTK), os autores concluíram que não houve diferença significativa para nenhum dos testes aplicados. Os resultados foram diferentes do presente estudo onde se observou diferença significativa por sexo na trave de equilíbrio estando semelhante a outras variáveis como o salto lateral, a transferência sobre plataforma e o salto monopedal.
No estudo realizado por Carminato (2010) com 230 crianças de 8 anos, de ambos os sexos, em que a normalidade para o teste KTK foi de ( $\mathrm{p}=0,11)$, os resultados mostram que a maioria das crianças $(42,5 \%)$ apresenta perturbação da coordenação motora, o que indica um baixo nível de desempenho motor. Esses dados foram semelhantes aos do presente estudo em que o nível de significância foi de ( $\mathrm{p}=0,756 ; \mathrm{p}=1,000$ e $\mathrm{p}=1,000$ respectivamente), inclusive, apontando mesmo escore entre os sexos tanto na transferência sobre plataforma quanto no salto monopodal.

O presente estudo demonstra baixo índice de desenvolvimento motor alcançado pelas crianças avaliadas 
pelo teste KTK, apesar da diferença significativa entre os sexos na trave de equilíbrio $(\mathrm{p}=0,002)$. Os demais testes não mostraram diferenças significativas referentes a comparação por sexo.

Para Gallahue, Ozmun (2005), essa fase motora especializada são padrões motores fundamentais maduros, em que a crianças já consegue formar habilidades motoras específicas e complexas, inclusive, passando para o estagio transitório.

A fase motora especializada é dividida em três estágios: o primeiro é o estagio de transição, que é caracterizado pela tentativa do individuo de aperfeiçoar ou combinar padrões motores maduros. O segundo estágio de aplicação (11 a 13 anos) e o terceiro de utilização permanente (14 anos) em diante. É quando ocorre um maior interesse nas crianças pelo esporte e nos padrões de desempenho. Estas se sentem atraídas por vários tipos de esporte e não sentem limitações fisiológicas, anatômicas ou ambientais. (GALLAHUE; OZMUN, 2005). Observou-se, no presente estudo, em relação ao desempenho nos testes, que nem todas as crianças entraram nessa fase de desenvolvimento.

Uma limitação desse estudo refere-se à quantidade de participantes, devido à dificuldade de devolução do TCLE com a assinatura do responsável. Uma quantidade maior de crianças poderia mostrar maiores diferenças na análise por sexo.

\section{Considerações Finais}

Então, de acordo com o estudo realizado, sugere-se que a disciplina educação física seja melhor direcionada pelos professores, para que sejam trabalhadas e bem desenvolvidas as habilidades motoras das crianças. Além disso, é necessário que as práticas motoras sejam valorizadas a ponto de dar liberdade para a criança se desenvolver com autonomia e naturalidade.

Conclui-se que, nos aspectos motores quanto à trave de equilíbrio, houve uma diferença significativa para os meninos na comparação por sexo. Porém em relação ao salto monopedal, saltos laterais e transferência sobre plataforma, não houve diferencia significativa por sexo, inclusive apontando mesmo escore entre os sexos tanto na transferência sobre plataforma quanto no salto monopedal.

\section{Referências}

BONAMIGO, E. M. R. et al. Como ajudar a criança no seu desenvolvimento. Porto Alegre: UFGRS, 1982.

CARMINATO, A. R. Desempenho motor de escolares através da bateria de teste KTK. 2010. 98f. Dissertação (Mestrado)-Departamento de Educação Física, setor de Ciências Biologica da Universidade Federal do Paraná, Londrina, 2010.

CRUZ, V. S. Desenvolvimento motor de crianças nos anos iniciais em uma escola pública de Lagamar dos Coqueiros. 2012. 51f. Monografia (Graduação) - em Educação Física do Programa UAB da Universidade de Brasília, Coromandel, 2012.

GallahuE, D. L; OZMUN, J. C. Compreendendo o desenvolvimento motor: bebês, crianças, adolescentes e adultos. São Paulo: Phorte, 2005.

GORLA, J. I; ARAÚJO, P. F; RODRIGUES J. L. Avaliação motora em educação física adaptada. São Paulo: Phorte, 2009.

KIPHARD, E. J.; SCHILLING, V. F. Köper-koordinations-testfürkinder: KTK.Weinhein: Beltz Test Gmbh, 1974.

MEINEL, K; SCHNABEL, H. Teoría del movimiento humano: motricidad deportiva. Buenos Aires: Stadium, 1988.

TANI, G. et al. Educação física escolar: fundamentos de uma abordagem desenvolvimentista. São Paulo: EPU/ EDUSP, 1988.

WEINECK, J. Manual do Treino Desportivo. 2. ed. São Paulo: Manole, 1986. 